\title{
Initiatives to Address Diversity, Equity, and Inclusion Within a Higher Education ABA Department
}

\author{
Jennifer Hilton ${ }^{1}$. Noor Syed ${ }^{1} \cdot$ Mary Jane Weiss $^{1} \cdot$ Lisa Tereshko $^{1}$. \\ Videsha Marya $^{1} \cdot$ Kimberly Marshall $^{1} \cdot$ Ksenia Gatzunis $^{1} \cdot$ Christen Russell $^{1}$. \\ Natalie Driscoll ${ }^{1}$
}

Accepted: 7 October 2021 / Published online: 25 November 2021

(c) Association for Behavior Analysis International 2021, corrected publication 2021

\begin{abstract}
Recent events have highlighted the need for behavior analysis to address issues of diversity, equity, and inclusion (DEI) in service provision and in higher education. There has been a call to action issued, noting the need for cultural humility and cultural responsiveness. An opportunity exists within training programs to ensure that students of behavior analysis are instructed in ways that promote cultural responsiveness and that equip them to serve diverse populations. Additionally, more needs to be done to engineer environments where students of behavior analysis are treated with respect and compassion, and to ensure that educational environments promote the comfort and success of all students. This article outlines the initiatives of an applied behavior analysis department to gather information about DEI on the local level, identify goals, implement change, and evaluate progress toward these goals.
\end{abstract}

Keywords DEI $\cdot$ Diversity $\cdot$ Equity $\cdot$ Higher education $\cdot$ Inclusion

Events that have taken place in the United States, including the deaths of George Floyd, Breonna Taylor, and Ahmaud Arbery, and the increase in incidents of violence and racism toward the Asian American and Pacific Islander community (Gover et al., 2020), have served as catalysts for social change throughout the United States and around the world. Given these instances, and the changes in demographics in the United States (Colby \& Ortman, 2015), it is imperative to continuously model, promote, and shape practices that advance equity and diversity.

There has been discussion within the field of behavior analysis about issues related to diversity, equity, and inclusion (DEI) in a number of contexts. When considering an assurance of DEI, it is important to evaluate the assortment of variables that compose an individual's culture, such as age, ethnicity, disability, gender

Mary Jane Weiss

mweiss@endicott.edu

1 Endicott College, Beverly, MA, USA 
identity, race, religion, sexual orientation, and socioeconomic status (Beaulieu et al., 2019). Each of these variables plays an important role when ensuring inclusion for all within a given community, including communities of behavior analysis. In one example of the evaluation of these variables, Jones et al. (2020) analyzed demographics reported by the Journal of Applied Behavior Analysis and found that demographic variables other than age and gender were often excluded from publications. Demographic information is crucial to understanding participants involved in research, particularly with regard to learning differences associated with diverse contextual variables. It is critical that our work in behavior analysis promotes inclusion and cultural responsiveness, which are practices that respect and honor diversity through the incorporation of cultural interests and preferences, and builds safe, inclusive, and respectful environments toward the promotion of justice and equity (Miller et al., 2019).

Large-scale change in the behavior of many individuals who are part of a coherent community (e.g., the field of behavior analysis) has been discussed in the literature on cultural systems analysis (e.g., Glenn, 1988, 2004; Glenn et al., 2016; Malott \& Glenn, 2006). Just as an individual's operant behavior is determined by consequences, cultural practices are determined by cultural outcomes, including the survival of the cultural system (Glenn, 1988). Cultural practices are responses exhibited by many members of a group and may generalize to novel members of the group or be passed down from generation to generation (Glenn, 1988). For example, a university could be a cultural system in which the members of the group, including faculty and students, engage in behavior that avoids directly discussing issues of DEI. A cultural practice might be using general examples in coursework that do not provide an opportunity for cultural considerations. To change cultural practices requires changing the interlocking behavioral contingencies (IBCs) that produce cultural outcomes. Glenn et al. (2016) defined IBCs as responses and their contingent reinforcement at the individual level. These are part of the metacontingency that maintains cultural practices. The second part of the metacontingency is the selection of the aggregate product of those IBCs in the environmental context (Glenn et al., 2016). In the example presented here, the university system could work toward increasing discussions about DEI through interventions (e.g., adding culturally diverse course content) designed to change the behavior of individuals within the system. These changed IBCs will maintain if they lead to reinforcement for the behavior of each individual member (e.g., professors' behaviors are reinforced by students participating more frequently in class). The change in the cultural practice (e.g., increased discussion of DEI by many members of the community) will be selected if the aggregate product or cultural outcome (e.g., more students enroll in the university program) increases the likelihood of the survival of the system.

With the recent calls for action to globally improve DEI, and specifically in the field of behavior analysis, some shifts in this cultural system have already begun to be observed. For example, the Behavior Analyst Certification Board (BACB) has released a new Ethics Code for Behavior Analysts (the "Code"), which will go into effect in 2022. This document emphasizes cultural diversity through the Code's Core Principles and Section 1 of the Code (BACB, 2020), as well as the supervision standards (Section 4 of the Code). These revisions are a step 
toward prioritizing DEI and may represent a cultural shift in the field of behavior analysis.

Despite changed practices, including the revised Code and the increased focus on DEI within conferences and publications, there are ongoing challenges and deficits in the field of behavior analysis. Authors have noted a lack of diversity among practitioners of behavior analysis and have called for improvements in the diversification of behavior-analytic professionals (Cirincione-Ulezi, 2020; Conners et al., 2019; Fong et al., 2017). To achieve this goal, we believe it is essential that DEI and culturally humble and responsive practices are incorporated directly into behavioranalytic coursework and supervised fieldwork (Beaulieu et al., 2019; Colby \& Ortman, 2015; Fong et al., 2017). According to demographic survey data collected by the BACB (n.d.), $56 \%$ of respondents identify as White, whereas only $21 \%$ identify as Hispanic/Latinx, $8 \%$ as Black, and $6 \%$ as Asian. Native American/Alaskan Native and Native Hawaiian/Pacific Islander each represented $0.3 \%$ and $0.6 \%$, respectively. Extrapolating from these data, it appears that the majority of our field identifies as White, which does not necessarily match an observed increase in the diversity of clientele whom behavior analysts serve. Implementing practices toward DEI and humility within our coursework and fieldwork, such as increasing access to materials, implementing antiracist curricula, acknowledging and addressing biases, and engaging in bidirectional feedback, may serve to attract an increasingly diverse body of behavior analysts and address systemic inequities. Using bidirectional feedback as a case example of how to address inequity, academic programs that solicit feedback from students early in their coursework may find that the students feel there is a lack of mentorship within the program by Black and Indigenous individuals and persons of color. Advisors and program coordinators can begin to address this immediately by engaging in the active recruitment of a diverse body of academic professionals. Simultaneously, the program may continue to work with students and solicit additional feedback with the goal of better understanding how to make coursework increasingly accessible and equitable (e.g., decreasing the cost of resources associated with classes and creating scholarship opportunities). These actions, driven by feedback from the students, may lead to sustainable change toward DEI within applied behavior analysis (ABA) and would not be possible without a feedback loop.

At this time, many higher education programs in behavior analysis do not include DEI and culturally humble and responsive practices in their curricula (Conners et al., 2019; Najdowski et al., 2021), despite increased diversity in higher education and the field at large. One contributing variable is the growth of online education, which has increased institutional reach to students in other countries (Collis, 1999; Sadykova \& Dautermann, 2009) and in geographically diverse locations in the United States. Some researchers have advocated for curriculum modifications that include multicultural information within the presented course materials and for varied instructional and assessment tactics to further support students of different cultures (Maringe \& Sing, 2014; Pincas, 2001). To build a community of DEI in higher education, DEI issues will need to be addressed in curricular development, supervised practice, professional skill development, and the recruitment of diverse individuals into the field. 
In the university system at Endicott College (where we are employed), it was determined that an assessment of DEI was essential. Given the issues occurring on national and international levels, and the call to action within the field of behavior analysis, the Department of Applied Behavior Analysis at Endicott College set about assessing the ways in which these issues could be understood "at home." About $18 \%$ of students in the ABA department live and practice outside of the United States. These students had frequently provided feedback that the existing ABA tools and resources were often Western-centered and not always culturally relevant to their current and future clinical work. Efforts had been made to identify these gaps, and some courses had been modified to incorporate more diverse materials and resources. We realized that these efforts needed to be broadly extended to other aspects of DEI, including the cultural variables of age, ethnicity, disability, gender identity, race, religion, sexual orientation, and socioeconomic status. The purpose of the current article is to document our attempts at assessing and subsequently addressing the needs of our faculty, staff, and students within the Applied Behavior Analysis Department at Endicott College in regard to DEI in order to create a climate of cultural responsiveness and social justice.

\section{Our Process}

\section{Formation of the DEI Task Force}

The process of evaluating and improving DEI in the behavior analysis program at Endicott College was initiated through the creation of the Cultural Diversity Task Force (the "task force"). The goal of forming the task force was to have a team of dedicated and concerned individuals that would drive the initiatives and take necessary actions. This and subsequent actions of the task force are summarized in Fig. 1. The task force was initiated and spearheaded by the leadership of the ABA program, which is led by two women. Leadership support has been shown to be important in progressing a culture of DEI; continued involvement from leadership is essential to successful change (Najdowski et al., 2021). The task force includes 10 other core members whose ages range from 33 to 57 years old. The task force includes people across varied nationalities, religions, races, socioeconomic statuses, orientations, and identifications. We have future plans to increase master's student participation.

As a preliminary step to guide the DEI work of the ABA program, the task force created a mission and vision statement as follows:

The mission of the Endicott College ABA program's DEI task force is to evaluate the current climate and enact actionable items towards meeting our values of a fully diverse, inclusive, and equitable higher education program. We strive to create a brave space that facilitates respectful dialogue regarding complex and difficult issues related to diversity, equity and inclusion in our department and our larger community. In pursuit of these goals, we will engage in ongoing data collection and analysis to identify needs, measure progress toward goals, and promote equity for all students, faculty, and staff. 


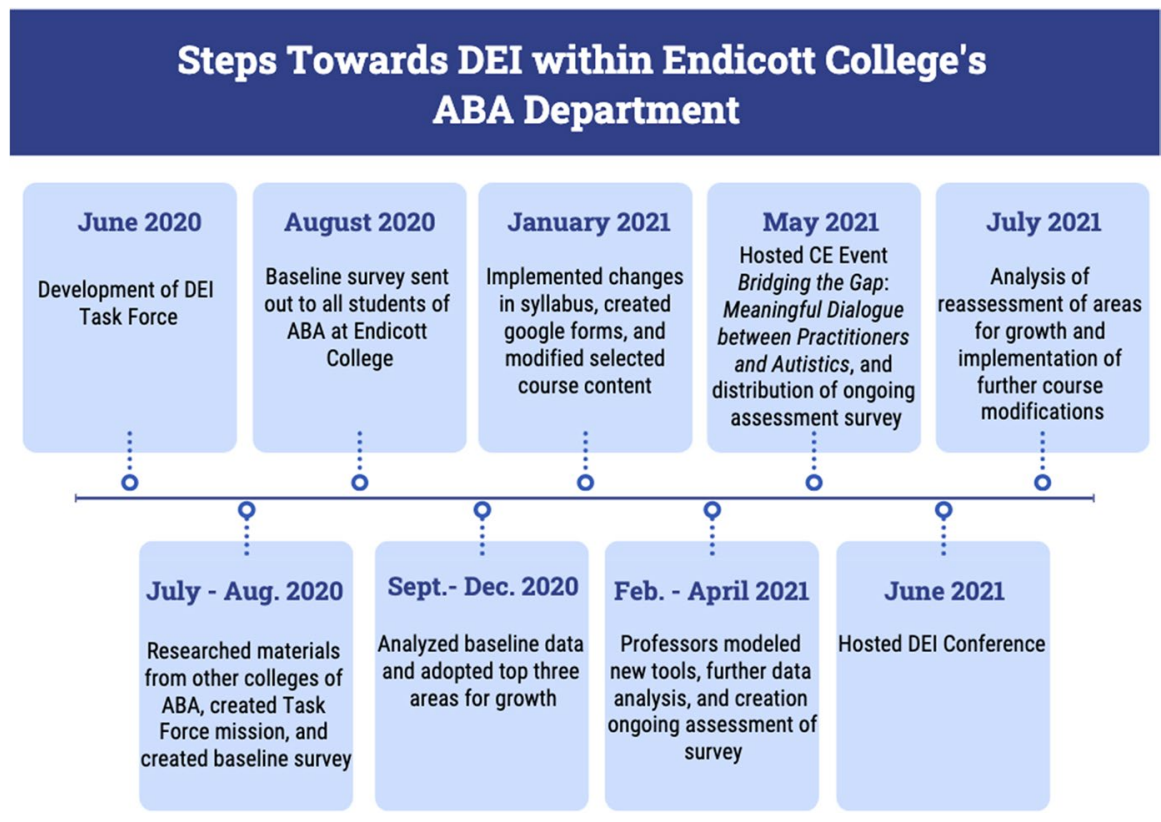

Fig. 1 Timeline of DEI process in the department of behavior analysis at Endicott College. Note. DEI = diversity, equity, and inclusion; $\mathrm{ABA}=$ applied behavior analysis

The vision driving the task force "is to continuously model, promote, and shape practices to advance equity and diversity, creating a climate of cultural responsiveness and social justice within the fields of behavior analysis and higher education."

\section{Systematic Analysis and Implementation of Change}

In order for cultural change to happen, a systematic process is essential to identify gaps and inform areas of needed intervention. Harvey (2017) identified seven steps in the development of intercultural curricula:

1. Identify important situational factors

2. Conduct a needs assessment

3. Identify/define key learning objectives

4. Decide upon feedback and assessment methods

5. Choose the most appropriate teaching and learning activities

6. Integrate activities into a coherent whole

7. Reflect on the process and identify key learning. (pp. 116-117) 


\section{Step 1: Identify Important Situational Factors}

The lack of DEI in the field of behavior analysis and higher education and precipitating events such as the murder of George Floyd were identified as important situational factors driving the needed change.

\section{Step 2: Conduct a Needs Assessment}

The task force conducted a needs assessment by creating and implementing a survey for completion by students in the master's and doctoral programs. Following exemption from the Endicott Institutional Review Board, an anonymous, voluntary survey was conducted via Qualtrics and distributed via email in the summer of 2020. Every question was optional, allowing students to answer those questions to which they felt comfortable responding. The first set of questions on the survey were designed to gather baseline information on the diversity of the student population. These questions included demographic and cultural information including race, ethnicity, socioeconomic status, gender, sexual orientation, disability, political beliefs, the program in which they were enrolled, and the number of semesters completed. Each of these questions was open ended, allowing for the students to self-identify.

The remaining sections were used to gather further information about current attitudes and behaviors regarding DEI within the department. A set of questions assessed the students' feelings of respect, empathy, and compassion across cultural variables in regard to student, faculty, and staff behavior. Occurrences of specific behaviors that may increase DEI were also investigated, including professors providing and asking about preferred pronoun and name use, the accessibility and diversity of materials, accommodations for language barriers, and professor and advisor availability for questions. Each section provided the opportunity for the student to further comment on any question or add additional information, if they wished to do so.

The survey also assessed whether the student had personally experienced a negative situation within the department at Endicott College. Specifically, it asked if they experienced or witnessed racism, sexism, homophobia, ageism, or any other prejudice. The survey additionally inquired as to whether students knew how to report an incident and whether they would feel comfortable doing so if an incident were to occur.

The survey was sent to 445 students, and 73 (16.4\%) students completed at least one question on the survey. This sample is representative of the broader community of students in graduate ABA programs at the college and was a relatively heterogeneous group, with $67 \%$ of participants identifying as White, $66 \%$ identifying as middle class, $78 \%$ identifying as female, and $71 \%$ identifying as heterosexual (see Table 1). More variability was observed in participants' selfreport of disability, as well as religious and political beliefs. Although most participants identified as abled or neurotypical (49\%), 5\% of students self-reported anxiety, $4 \%$ of students self-reported ADD/ADHD, and $4 \%$ of students selfreported depression. In regard to political beliefs, the plurality of participants identified as independent (33\%), whereas others identified as Democratic (19\%) or as not having a political belief (11\%). Finally, in relation to religion, students 
Table 1 Demographics of survey participants

\begin{tabular}{|c|c|}
\hline Demographic variable (total responses) & Frequency of responses \\
\hline Race $(n=66)$ & $\begin{array}{l}\text { White (49); Asian (5); Black (3); human (2); African (1); } \\
\text { African American (1); American (1); Asian/White (1); Black/ } \\
\text { White (1); Chinese (1); multiracial (1) }\end{array}$ \\
\hline Ethnicity $(n=86)$ & $\begin{array}{l}\text { White (37); American (18); Irish (6); Asian (3); European (3); } \\
\text { African (2); French Canadian (2); Hispanic (2); Portuguese } \\
\text { (2); Black (1); Chinese (1); Filipino (1); German (1); Haitian } \\
\text { (1); Indian (1); Latino (1); Italian (1); Native American (1); } \\
\text { Scottish (1); Swedish (1) }\end{array}$ \\
\hline Socioeconomic status $(n=55)$ & Middle class (48); lower class (7) \\
\hline Gender $(n=65)$ & Female (57); male (6); gender fluid (1); don’t identify (1) \\
\hline Sexual orientation $(n=60)$ & $\begin{array}{l}\text { Heterosexual (52); gay (3); asexual (2); pansexual (1); don't } \\
\text { identify (1) }\end{array}$ \\
\hline Disability $(n=53)$ & $\begin{array}{l}\text { Abled/neurotypical (36); anxiety (4); ADD/ADHD (3); depres- } \\
\text { sion (3); learning disorder (2); auditory processing disorder } \\
\text { (1); autism (1); bipolar disorder (1); OCD (1); neurodivergent } \\
\text { (1) }\end{array}$ \\
\hline Political beliefs $(n=61)$ & $\begin{array}{l}\text { Independent (24); Democrat (14); none/neutral (8); Republican } \\
\text { (4); liberal (3); progressive (3); moderate (2); socialist (2); } \\
\text { realistic (1) }\end{array}$ \\
\hline Religion $(n=58)$ & $\begin{array}{l}\text { Catholic (15); Christian (12); agnostic (7); none (6); athe- } \\
\text { ist (5); Jewish (3); spiritual (3); Presbyterian (2); Buddhist } \\
\text { (1); Episcopalian (1); interfaith (1); Methodist (1); secular } \\
\text { humanist (1) }\end{array}$ \\
\hline
\end{tabular}

Note. All participant demographic labels used are consistent with what was reported by the participants. Participants were not limited to one label per demographic variable and were able to fill in responses

self-identified as Catholic (21\%), Christian (16\%), agnostic (10\%), and none $(8 \%)$; the remainder did not report an affiliation.

Participants generally agreed or strongly agreed that students in the ABA program were respected at Endicott regardless of their demographic characteristics (range 56\%-74\%). Ratings at the lower end of this range related to respect based on political beliefs $(56 \%)$ and religious beliefs (61\%). A large percentage (82\%) of students agreed or strongly agreed that professors were empathic and compassionate toward them. The majority of participants also agreed or strongly agreed that other individuals at the university (e.g., advisors, other university staff, and students) were empathic and compassionate toward them. Students endorsed similarly positive ratings for feeling respected by individuals from these categories. Table 2 summarizes the results of this question about general levels of respect for diverse groups and includes additional information about the extent to which students believed persons of their identity characteristics were consistently respected. Results regarding respectful interactions were high, both for general impressions and for identity-specific responses.

It was important to obtain information about negative experiences among students, particularly in the area of discriminatory and inequitable behavior (see 


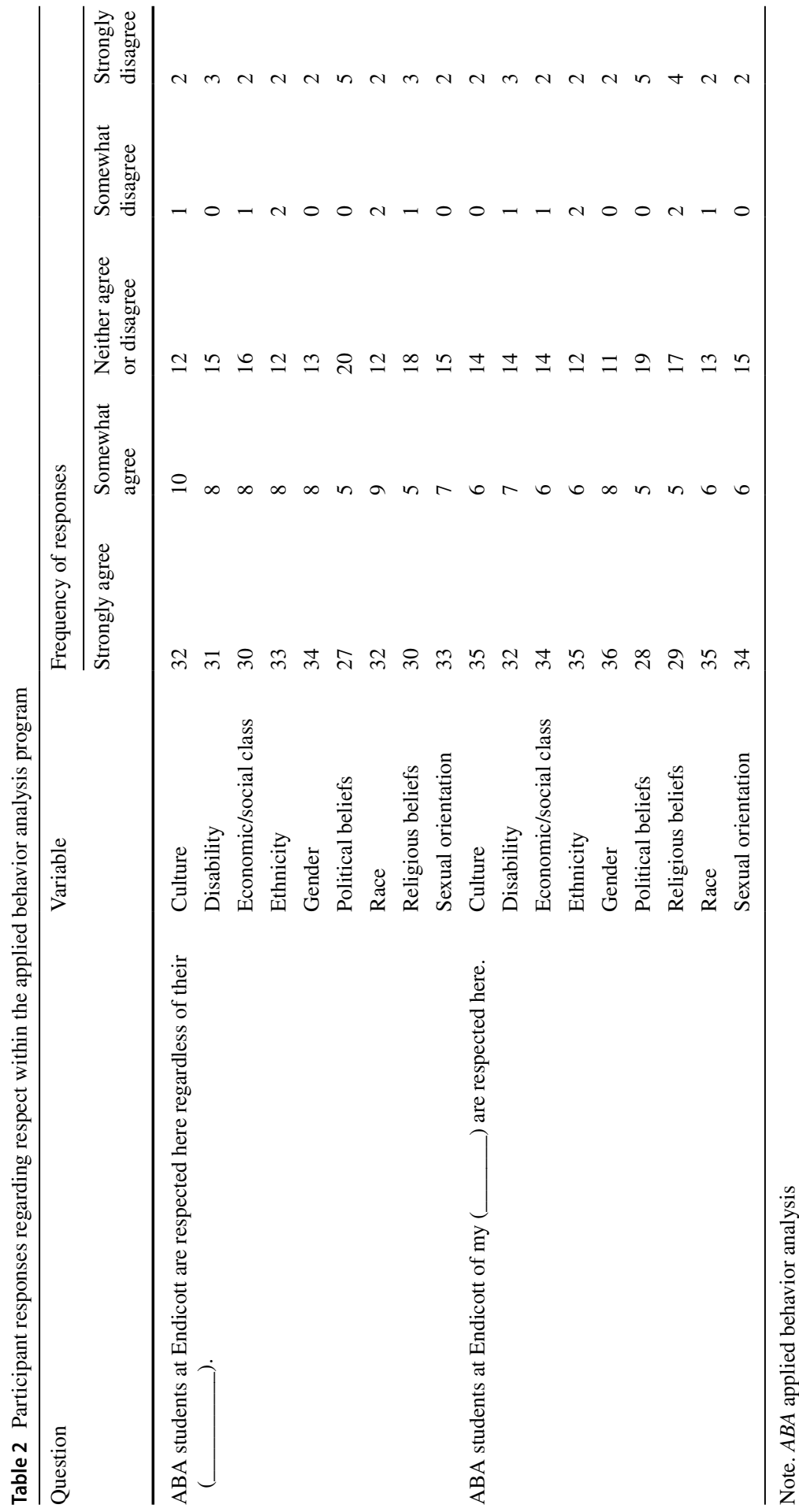


Table 3). Out of the 57 students who responded to the question "I have experienced racism, sexism, homophobia, and/or other prejudice by advisors at Endicott College," 56 students reported that this had never occurred, and one student responded that this had occurred multiple times. Prejudicial experiences were also assessed for interactions with professors, fellow students, and college staff. One student reported that they had experienced prejudice one or more times with professors, zero students reported experiences with other staff members (e.g., library staff, bursar's office staff), and four reported that prejudice had been experienced multiple times from other students at Endicott. Although the majority of the students who completed the survey did not indicate adverse experiences, the opinion of the task force is that even one instance is too many.

The next set of survey questions about current attitudes and behaviors regarding DEI within the department was used to determine the immediate and future goals of the task force. These goals centered on the mission of broadly improving diversity and equity at Endicott and decreasing the likelihood that students would encounter adverse experiences in the future. Importantly, most students reported that they felt comfortable reporting experiences of prejudice to someone within the Endicott community (73\%); however, few reported knowing how to start the process (30\%). Additionally, there were high percentages of situations in which faculty failed to provide an inclusive environment; for example, many students reported that they were never asked about their preferred name (40\%), they were never asked about their pronouns (78\%), or the professor failed to share their own pronouns (50\%). (See Table 4 for this information.) Another noted concern was that $26 \%$ of students disagreed or strongly disagreed that materials and coursework adequately covered important cultural considerations related to behavior analysis (see Table 5). As a result of the survey, actionable items were created, with timelines to promote expediency, to address all areas that were reported as problematic.

\section{Step 3: Identify/Define Key Learning Objectives}

Following the needs assessment, immediate priorities were identified. These priorities included providing professors with training and resources to improve the student experience. Google Forms were created for professors to share with their students at the start of each course to give them an opportunity to share pronouns and preferred names, as well as preferences and needs with regard to coursework (e.g., international access to readings, access to recorded lectures). In addition, students could provide immediate feedback to the professor about the course throughout the semester (Appendices 1 and 2).

\section{Step 4: Decide Upon Feedback and Assessment Methods}

The Google Forms offered multiple ways for students to provide feedback on identified key learning objectives. Students were also provided with information about how they could report adverse experiences, such as submitting a summary of the incident with preferred ways to address it, to their course instructor or to advisors of the program (Appendices 2 and 3). All professors were instructed to include these 


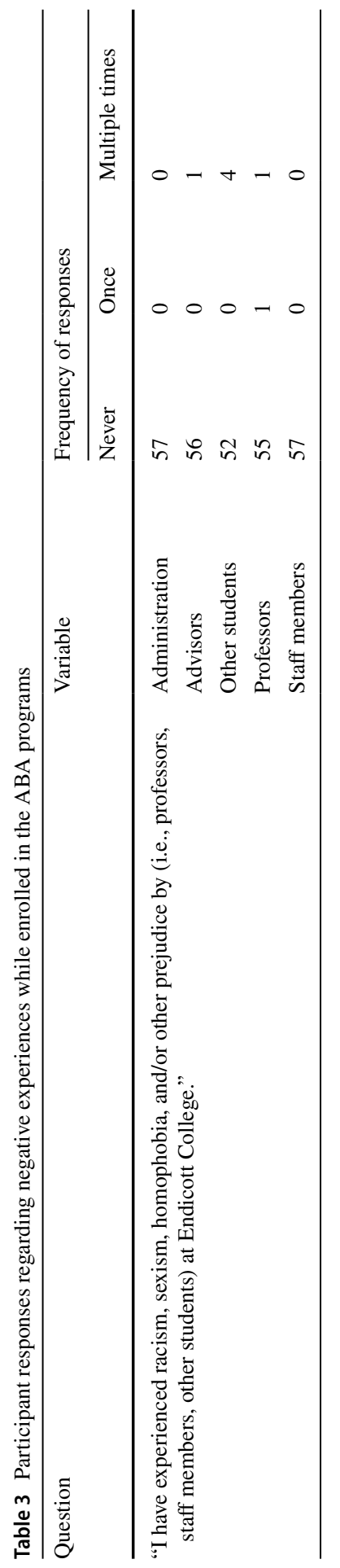


Table 4 Participant responses regarding appropriate pronoun and name use

\begin{tabular}{|c|c|c|c|c|c|c|}
\hline \multirow[t]{2}{*}{ Question } & \multirow[t]{2}{*}{ Variable } & \multicolumn{5}{|c|}{ Frequency of responses } \\
\hline & & Always & $\begin{array}{l}\text { Most of the } \\
\text { time }\end{array}$ & $\begin{array}{l}\text { About half } \\
\text { the time }\end{array}$ & Sometimes & Never \\
\hline \multirow{2}{*}{$\begin{array}{l}\text { My professors } \\
\text { ask about my } \\
(\square\end{array}$} & Preferred name & 9 & 4 & 5 & 15 & 22 \\
\hline & Pronouns & 2 & 3 & 1 & 6 & 43 \\
\hline $\begin{array}{l}\text { My professors } \\
\text { share their } \\
\text { ( }\end{array}$ & Pronouns & 6 & 2 & 3 & 16 & 27 \\
\hline
\end{tabular}

methods of reporting on their course sites. Finally, the task force created a brief version of the original survey to be sent out at the end of each semester to continually evaluate the impact of these actionable items on the master's and doctoral students in the ABA program.

\section{Step 5: Choose the Most Appropriate Teaching and Learning Activities}

Interventions were built to directly address those key learning objectives identified through the survey. For example, core coursework was revised in order to weave in culturally diverse materials and cultural considerations. These and other data-driven interventions will be discussed in further detail in what follows.

ABA Course Content Initiatives The task force reviewed resources to build its understanding of how the instructional environment could be altered to increase the level of inclusivity. A primary, initial goal was the revision of syllabus language. The principal resource used to update course syllabi was developed by the Harriet W. Sheridan Center for Teaching and Learning (n.d.) at Brown University. As noted previously, a sample statement was created for instructor use and was included on syllabi for every course in the ABA Department (master's and doctoral level). The goal for the sample syllabus statement was multifold; the first goal was to promote respectful discourse within the courses and provide ways to report and resolve any racist, homophobic, sexist, ageist, ableist, or otherwise discriminatory and inequitable behavior. A second goal was to deliver a strong message about the departmental commitment to inclusivity by affirming its dedication to and prioritization of practices promoting DEI. Instructors were encouraged to reflect their own beliefs and values by individualizing the message in the syllabi about their personal dedication and commitment (Appendix 3).

Additional changes were made in the following areas: (a) encompassing culturally responsive course content within core and elective courses (both synchronous and asynchronous formats; Table 6), (b) providing additional resources, (c) selecting culturally diverse readings, (d) modifying study guide questions, (e) presenting scenarios that illustrate examples and nonexamples of culturally humble behavior, (f) updated lectures, (g) using diverse names in examples, and (h) incorporating other 


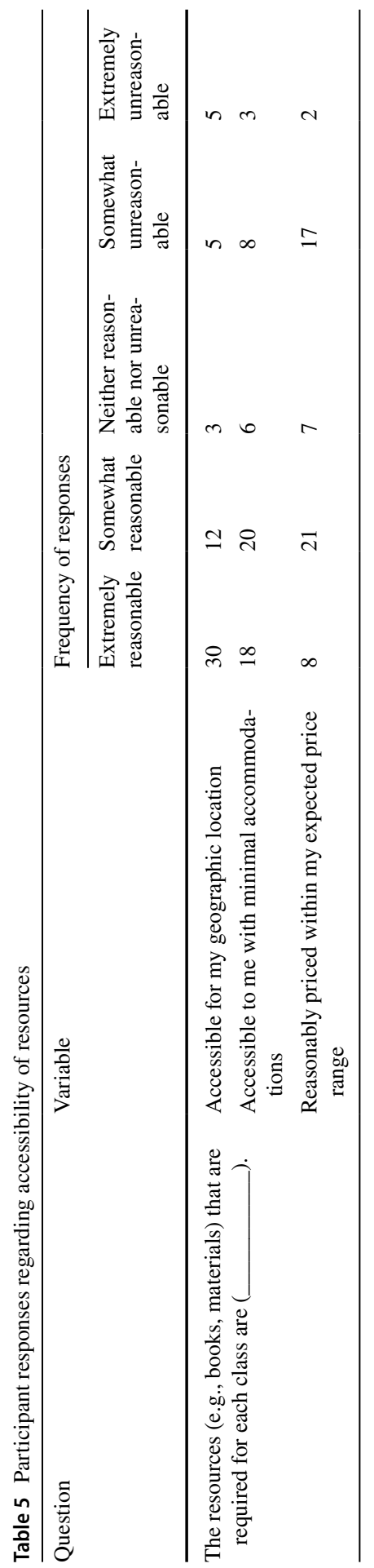


Table 6 Definitions of Relevant Terms

\begin{tabular}{|c|c|}
\hline Term & Definition \\
\hline Cultural variables & $\begin{array}{l}\text { The assortment of variables that compose an individual's culture, such as age, } \\
\text { ethnicity, disability, gender identity, race, religion, sexual orientation, and } \\
\text { socioeconomic status }\end{array}$ \\
\hline $\begin{array}{l}\text { Discriminatory and } \\
\text { inequitable behavior }\end{array}$ & $\begin{array}{l}\text { Occurs when students, faculty, or staff are treated in a way that is different from } \\
\text { their peers as a result of some aspect of their identity (race, socioeconomic } \\
\text { status, culture, ethnicity, gender identity, sexual orientation, disability, politi- } \\
\text { cal beliefs, religious beliefs, etc.; Pager \& Shepherd, 2008) }\end{array}$ \\
\hline Diversity & $\begin{array}{l}\text { The practice of including or involving people across cultural variables (e.g., } \\
\text { age, disability, ethnicity, expression, gender, orientation, race, socioeconomic } \\
\text { status) }\end{array}$ \\
\hline Equity & $\begin{array}{l}\text { The management of all institutional policies and procedures, and individual } \\
\text { behavior that is free from bias or favoritism and is based on individualized } \\
\text { context }\end{array}$ \\
\hline Inclusion & $\begin{array}{l}\text { The practice of incorporating individuals across all cultural variables through } \\
\text { equal access and accommodations }\end{array}$ \\
\hline Cultural humility & $\begin{array}{l}\text { A lifelong commitment to self-evaluation; focuses on continual self-reflection, } \\
\text { the recognition of power imbalances, and the development of partnerships } \\
\text { within communities (Tervalon \& Murray-Garcia, 1998; Wright, 2019) }\end{array}$ \\
\hline Cultural responsiveness & $\begin{array}{l}\text { Congruent behaviors, attitudes, and policies that are consistent with apprecia- } \\
\text { tion and respect for diversity, equity, and inclusion, including (a) assessing } \\
\text { and understanding one's own experiences and biases; (b) learning from } \\
\text { and appreciating the experiences of others and viewing their differences as } \\
\text { strengths; (c) implementing practices and tailoring systems to acknowledge } \\
\text { and honor the diverse backgrounds of individuals; (d) creating safe, inclusive, } \\
\text { and respectful environments; and (e) addressing inequities related to identity } \\
\text { through the promotion of justice and equity (LeBlanc et al., 2020; Miller } \\
\text { et al., 2019; Wlodkowski \& Ginsberg, 1995) }\end{array}$ \\
\hline Synchronous courses & $\begin{array}{l}\text { Held online in real-time wherein students sign on at a specific time in order to } \\
\text { attend class via video software, such as Zoom, for specific times each week, } \\
\text { and interact with other students and the professor live through this video } \\
\text { platform (Finol, 2020) }\end{array}$ \\
\hline Asynchronous courses & $\begin{array}{l}\text { Held online, but do not have a specific time that students have to sign in to } \\
\text { attend class; students access their course materials on the course website } \\
\text { and hand in their materials by the assigned deadlines, and interact with other } \\
\text { students and their professors via email and asynchronous discussion forums } \\
\text { (Finol, 2020) }\end{array}$ \\
\hline
\end{tabular}

Note. The terms in the table are used throughout the article. Definitions of these terms are provided to orient the reader to how they will be used

course-specific elements. To achieve the first goal of infusing culturally responsive course content, student assignments involved creating and enhancing instructional material. These additional assignments equip students with skills for integrating cultural responsiveness into teaching, clinical practice, and research. Guest lectures offered in the courses included parents and professionals from across the globe who provided diverse perspectives on a multitude of relevant issues. At the master's level, guest lecturers (national and international) specific to cultural responsiveness and DEI were incorporated into certain courses (e.g., Ethics, Effective Collaboration). 
At the doctoral level, a synchronous course on cultural humility/DEI is offered as an elective to the students in the program and adjunct professors of the department.

Another change included refining discussion forums in the asynchronous classes. For example, a specific introduction discussion forum was added for Week 1 within each course. In this discussion forum, the instructor has the choice of sharing their pronouns and preferred name, as well as any other information specific to the instructor's professional background and interests. Students are encouraged to introduce themselves in an effort to promote familiarity among one another and with the instructor. For subsequent discussion forums, the task force encouraged instructors to infuse relevant DEI content into their courses in the form of offering discussion prompts, sharing articles, and posting links to related material. The function of these changes was to increase representation across all cultural variables within discussion forums (Appendix 4).

Future initiatives include (a) expanding DEI trainings; (b) increasing cultural humility readings across courses; (c) inviting guest speakers with diverse backgrounds and identities to additional and elective courses; (d) implementing behavioral skills training in all master's-level classes to teach compassionate care, cultural responsiveness, and ethical decision making; (e) developing global learning outcomes for all courses; (f) incorporating DEI content into student and staff orientations and ongoing training; (g) continually assessing student expectations of the task force; and (h) shaping Writing Center and tutoring services to be more inclusive and ABA specific. Future considerations for international students include (a) inclusion for students from different geographic locations, (b) access to materials/readings for those who have limited access (e.g., international shipping issues, governmental restrictions on specific websites or resources), (c) resources for students who are English language learners, and (d) resources for students who may not have the financial means to purchase materials (e.g., specific software that cannot be used on a Chromebook or iPad).

Course Illustration One specific example of a course that was updated is Behavioral Assessment, a master's-level course that focuses on functional behavior assessments (FBAs), preference assessments, and skills/needs assessments. From the 1st week of the semester, students are introduced to the importance of considering and respecting cultural norms and differences throughout the entire assessment process. They are assigned two readings (i.e., Moreno et al., 2014; Moreno \& Gaytán, 2013) and are required to write a response regarding the importance of considering culture within the FBA process, including a discussion of the changing demographics within the United States, current issues regarding the disproportionate use of disciplinary actions with students from different races, recommendations/strategies for decreasing educator biases and other issues, and an application to the students' own clinical work. Students are also encouraged to share their own personal experiences of working with clients from different backgrounds within the weekly discussion boards. Furthermore, when they begin their FBAs, they must justify the cultural relevance and appropriateness of the target behaviors selected using relevant literature (e.g., Fong et al., 2016). For students who may not have access to clients during the course (e.g., due to the COVID-19 pandemic), information regarding a hypothetical 
client for the FBA is given, including various cultural factors for the students to consider (e.g., language used in the home, family values, attendance of religious activities). Additional course topics include (a) varying perspectives regarding causes of autism spectrum disorder, (b) differences in levels of acceptability for various medical and behavioral treatments, (c) how the language used during functional analysis may impact the results with individuals who are multilingual (e.g., Rispoli et al., 2011), and (d) ways to consider the cultural relevance of aspects of existing assessment tools, such as the Verbal Behavior Milestones Assessment and Placement Program (VB-MAPP). Lastly, each student is presented with a scenario in which a behavior analyst interviews a family whose primary language and culture differ from their own. Upon analyzing the scenario, students should identify that the behavior analyst did not assess the family's preferred language prior to the interview, disapproved of another child functioning as the interpreter during the interview despite the family's request, and denied the involvement of the extended family during the interview, which did not respect the family structure and dynamics.

Bidirectional Communication Another area of need identified by the ABA program at Endicott College centered around creating a way for students to provide initial information and ongoing feedback to their instructors and the department as a whole throughout their tenure with the program. As a means for students to provide information and feedback, the following forms and surveys, created using Google Forms and Qualtrics, are used: (a) Getting to Know You: Start of Semester Survey, (b) Learning \& Support Feedback, (c) Embracing a Culture of Change: Instructor, (d) Embracing a Culture of Change: Program Advisors. All of the aforementioned surveys are provided to students at the beginning of the semester; however, it is not mandatory that students respond to individual questions on the survey or to the survey as a whole. These surveys ask students to provide information regarding their personal background, experiences within the program, and learning preferences. They also provide a means for students to give faculty and staff confidential feedback about progress made toward DEI initiatives, as discussed in the fourth step of Harvey's (2017) recommendations.

The Getting to Know You: Start of Semester Survey (Appendix 1) provides students with the opportunity to share information, such as preferred names and pronouns, with each of their instructors. The Learning \& Support Feedback form (Appendix 2) gives students the opportunity to share what they are finding helpful within a course, as well as indicate what can be improved. Engaging in this dialogue at the onset of a class will help the instructor better understand how to serve their students while ideally empowering the students' voices to share teaching methodologies that are helpful to their growth. The Embracing a Culture of Change: Instructor survey (Appendix 5) provides a method to report situations in which students felt discomfort or offense surrounding cultural identifications, microaggressions, equity, and social injustice. There is also another version of this form that is shared with program directors only. This survey uses the same questions but allows students to share experiences that they may not feel comfortable sharing directly with their instructor. Students can choose to use this form should they wish to report a situation that occurred with an instructor, an advisor, a staff member, or fellow students. 
Finally, the task force created a brief version of the original survey to be sent out at the end of each semester to continually evaluate the impact of these actionable items on the master's and doctoral students in the ABA program.

Our goal is to create a culture in which students feel comfortable sharing reports of possible inequity in order to achieve a respectful resolution. Power imbalances are often associated with systemic oppression and social injustice (Gillborn, 2018); therefore, we are seeking to empower students to have a stronger voice in responding to any potentially problematic situations with their peers or with faculty and staff. This methodology also allows advisors and instructors to respond quickly, engaging in conflict resolution and systemic change as appropriate.

Professional Development for Faculty Once initiatives were defined and interventions had been incorporated into the coursework, information was shared with all faculty members about changes in course content, instructor interactions, and bidirectional communication with students. A mandatory department-wide meeting was held to summarize the new expectations. The program director emphasized the foundational values of the department, its short- and long-term goals, and its vision for increasing inclusion within the department. This training is now embedded into the initial semester onboarding for all instructors.

The task force was introduced at the meeting, and its intent was shared, along with results from the initial needs assessment. Department-wide initiatives that required the participation of all faculty were explained, as well as the rationale for their universal application. Specific topics discussed included updated syllabi, course content, survey results, task force priorities, student engagement, introductory survey forms, and resources. Samples of course changes were shared with faculty to illustrate how culturally responsive content could be infused into the core courses. In addition to the department-wide meeting, supplementary training programs provided as part of college-wide initiatives were made available for faculty. One such training was the Safe Zone Project, which is a workshop to promote LGBTQ+ awareness, as well as to educate and train allies (The Safe Zone Project, n.d.). In addition to faculty training, the ABA department organized their first annual Cultural/DEI Conference, held in June 2021.

To drive long-term success and the continued evolution of the departmental initiatives, it is important to continually evaluate interventions and target the maintenance of skills within the cultural system. To promote systemic change, it is important to embed training on skills related to intercultural responsiveness across coursework (Beaulieu et al., 2019), develop standards for practice and competency, and create ongoing opportunities for continuing education related to DEI (Fong et al., 2016).

\section{Step 6: Integrate Activities Into a Coherent Whole, and Step 7: Reflect on the Process and Identify Key Learning}

The department is currently in the process of Steps 6 and 7 of Harvey's model. Some of these initiatives include implementing and reviewing follow-up surveys, 
inviting master's-level students to join the task force, and extending the effort to include intercultural information in all courses, including electives. In addition, the task force is working with the college's DEI leadership to further refine and integrate approaches to enhance the DEI initiatives, and to focus on belonging (DEI-B) as an additional outcome. The concept of belonging has been added at the college level to ensure that there is an assessment of the extent to which students and faculty feel a part of the college as a whole, feel welcomed by the institution, and feel connected to others within the college.

\section{Conclusion}

Contingency management at the cultural level, even at a small scale (e.g., a university setting), requires organized systems to identify targets for change, implement interventions designed to change IBCs, monitor shifts in cultural practice, and continue to analyze and respond to these changes. The departmental momentum is most certainly enhanced by the intense efforts at the college to address DEI-B issues broadly. Faculty training, with a focus on engagement and action, is increasing the salience of these issues and changing the conversations in faculty and staff forums. These sentiments were echoed in Harvey's (2017) recommendations, in which the final step describes the importance of reflection and learning from the process of engaging in systems change through the development of intercultural curricula. Understanding the needs of a system, including those individuals for whom intervention will be most important, is a necessary condition for success.

The cultural climate across the globe, as well as within smaller communities, has included instances that have highlighted the importance of having active conversations centered around DEI. As discussed earlier, a critical component in creating equity within coursework is the bidirectionality of feedback. For example, soliciting feedback regularly throughout the course allows the faculty to make changes in real time, rather than waiting for a subsequent semester. Our objective in creating these surveys was to give students a way to communicate learning supports that would increase the efficacy of course content and allow students to have greater control of their own learning and the manner in which they will be addressed. To engage in humble behavior as instructors, we argue that it is critical to create feedback loops throughout the semester in which students have the opportunity to share what they are finding helpful in a course's instructional strategies and to give suggestions regarding how content delivery can be improved. In addition, modeling bidirectionality and humility may help students learn through observation and implement this in their own practice and future work, precipitating a cultural shift toward equitable and culturally responsive behaviors.

It is also important to place this discussion in the context of the broader field of behavior analysis to determine how these behaviors can be maintained. Presently, the BACB has no continuing education requirements specific to cultural responsiveness or DEI. If a requirement for continuing education related to cultural responsiveness were added, then students of behavior analysis and certified behavior analysts may participate in education and training in these areas. The cultural system would 
shift to meet this demand, and, as such, the interrelated contingencies would support further development of such education and training. As with all behavior change, it is critical to consider how we can begin to shape our practices. Education, implementation, and research in our field should increasingly embed cultural considerations within our analyses. At the level of higher education, we should seek to include diverse voices in our curricula, evaluate the accessibility of our resources, speak with our students regarding how to increase inclusivity within the program, and create feedback loops that allow instructors and staff to continually evaluate practices as related to DEI. If behavior-analytic educational programs increased their focus on these behaviors, we posit that the field will accelerate the speed of increasing DEI within our research and practice.

Advancement in DEI in behavior analysis and within higher education is an evolutionary process. Organizational systems commonly consist of complex interlocking contingencies, and changes within the larger system can be challenging due to the nature of changing component macrosystems (Wilson et al., 2014). A closer examination of these smaller systems, however, assists in the identification of steps to be initiated that will lead to a greater systemic change. Although shifting individual contingencies within a higher education environment may lead to cultural change (e.g., one instructor sharing their pronouns may cause another to do so; Glenn, 1988, 2004), group interventions may be more economical in cultural evolution. The ABA department at Endicott College decided to implement interventions both at the individual and at the group levels within our own smaller system (e.g., forming language centered around DEI in coursework syllabi for all instructors to adopt). In starting with recurring interlocking contingencies over which we had control, our objective remains that these departmental changes will function to affect broader metacontingencies in behavior analysis. We hope our actions will evoke establishing operations and subsequent reinforcement contingencies for other behavior-analytic graduate programs to advance cultural change toward DEI.

\section{Appendix 1}

\section{Getting-to-Know-You Form}

Hi everybody! I would like to invite you to let me know your preferred name, pronouns, and other information about things that would help you with success in this course.

- My full name as it appears on my class registration is (for administrative purposes).

- I prefer to be called (does not have to match Endicott records).

- My pronouns are

- If assignments require collaboration with other students, my preferred working style with peers is . (The instructor will do their best to match you with others who indicate similar preferences. All meetings will be scheduled at your discretion.) **Please note: This is not always applicable for your course.** 
A. Working primarily through collaborative tools, such as Google Docs, with very limited meetings

B. Working through collaborative tools, but scheduling ongoing meetings as necessary

C. Synchronous working meetings

D. Other:

- I am most looking forward to learning about in this course.

- I would feel the most supported in this course if

- Additional supports or resources that I might find helpful in learning during this course include (e.g., office hours, additional media links, periodic check-ins).

- Is there anything else you want me to know about you?

\section{Appendix 2}

\section{Learning and Supports Feedback}

The form states the following:

The ABA department at Endicott College strives to create a culture of mutual respect where students feel comfortable and supported in providing feedback to their instructors throughout the semester, not only at the end during student evaluations.

This feedback helps to improve our classes! While we cannot guarantee that all requested changes will be made, we do promise to review your feedback carefully and engage in modifications to the best of our ability. All responses are anonymous, and this form is not mandatory; if you wish to leave your name, however, you may do so at the end of the form.

The following language is used to introduce both forms. Underlined phrases indicate the text will vary according to each unique form.

Should a situation arise that causes discomfort or offense, academically or socially, please use this form to provide feedback and/or a summary of the situation. This form will go directly to (instructor of the course OR program advisors).

Unless you choose to include your name, this form will NOT collect your identifying information. If you choose to include your name, we will follow up with you as soon as possible.

ALL RESPONSES ARE AND WILL BE KEPT CONFIDENTIAL. This form will be deleted upon review by the (instructor of the course OR program advisors).

The full text of questions is as follows:

1. Required: Please provide a detailed summary of the situation. We ask that you provide sufficient information to allow for us to mitigate, address, and/or take measures to further prevent this from occurring. The details may or may not include the names of others (students, faculty, staff). Kindly proceed in how you 
are most comfortable. To the extent possible, we will never intentionally reveal your identity. For example, you may be identifiable based on the nature of the situation, but we will protect your confidentiality in every possible way.

2. Required: Ideally, how would you like the situation to be resolved? Responses may include, but are not limited to, a mediated conversation with specific colleagues/faculty/staff, open discussion in class addressing the issue broadly without identifying information, and conversation involving only specified colleagues/ faculty/staff and leadership.

3. Not required: If you feel comfortable, please share any additional information that might help us to understand when and where this situation occurred (e.g., course name, study group).

4. Not required: If you would like us to follow up with you directly, please leave your name and contact information. Your contact information will not be shared with others.

\section{Appendix 3}

\section{Syllabus Statement}

The Applied Behavior Analysis Program at Endicott College seeks to support students from all backgrounds and perspectives, and demonstrate respect for cultures, identities, and diverse learning styles. Diversity in our student body is a strength. Resources utilized in our courses are selected to benefit all involved. It is our intent to present materials and activities that are respectful, inclusive, and embrace diversity within, but not limited to, gender identity, sexuality, disability, socioeconomic status, ethnicity, race, nationality, and religion. Our goal is to create a culture of acceptance and comfort in expressing oneself within and outside the classroom. Your suggestions regarding how we can improve the inclusivity of our program are encouraged and appreciated.

Please note that it is the intention of this course and program to create an educational environment where teachers and students can freely discuss their thoughts and opinions about the material that is covered. Due to the nature of some of this material, it is likely that you will encounter others with differing views from your own. It is the expectation that all thoughts and opinions will be honored and respected, regardless of agreement or disagreement. If a situation ever arises that causes discomfort or offense, please do not hesitate to let your professor, advisor, and/or program director know. If you experience difficulty with your professor or a fellow student, you are encouraged to approach the professor directly. If you would like to raise the issue to the class, your professor will support you in this endeavor. You can also do one or more of the following:

- Contact the Executive Director of Programs in ABA (name and email) or the Director of Programs in ABA (name and email).

- Fill out the anonymous feedback form in order to notify your professor of any concerns you may have. 
- Approach a trusted advisor, former professor, fellow student, or other individual you feel comfortable with, who can assist you in raising the issue with the professor or other members of the department.

- Approach the designated Title IX officer at the university (name and contact information).

(This is a model and should be personalized by the instructor of the course.) Along with many other people, I am currently in the ongoing process of improving my understanding and knowledge of diverse perspectives and identities. I am receptive to feedback if I or anyone else within the course says or does anything to make you feel uncomfortable for any reason. If you feel comfortable, you may contact me directly via email or Canvas messenger. In addition, at any given time, there are many life events that may be impacting you and require adjustment and flexibility.

- If you would like to submit anonymous feedback, you can do so using this Google Form. Here is the link to the template (include link; instructors will need to slightly modify), which will collect your response without any identifying information.

My preferred name is and my pronouns are I would like to use your correct name and pronouns in class and correspondence. In case I make an error, please correct me. There is also a form that I will post at the start of the course that will provide an opportunity for you to share important information.

\section{Appendix 4}

\section{Sample Discussion Forum Prompts}

- Talk about the attached contemporary issues article or the week's readings: Fong et al. (2016). Developing the cultural awareness skills of behavior analysts. Behavior Analysis in Practice, 9(1), 84-94.

- Please make an original post about the attached contemporary issues article: Melendez et al. (2021). Practical resources for talking to children with autism about systemic racism. Behavior Analysis in Practice, 14, 451-461.

- Please make an original post about the attached contemporary issues article: Leland and Stockwell (2019). A self-assessment tool for cultivating affirming practices with transgender and gender-nonconforming (TGNC) clients, supervisees, students, and colleagues. Behavior Analysis in Practice, 12(4), 816-825.

- Please make an original post about the attached contemporary issues article: Pritchett et al. (2020). Social justice is the spirit and aim of an applied science of human behavior: Moving from colonial to participatory research practices. 


\section{Appendix 5}

\section{Feedback Form: Embracing a Culture of Change}

Should a situation arise that causes discomfort or offense, academically or socially, please use this form to provide feedback and/or a summary of the situation. This form will go directly to the instructor of the course.

Unless you choose to include your name, this form will NOT collect your identifying information. If you choose to include your name, we will follow up with you as soon as possible.

ALL RESPONSES ARE AND WILL BE KEPT CONFIDENTIAL. This form will be deleted upon review by the instructor.

Please provide a detailed summary of the situation. We ask that you provide sufficient information to allow for us to mitigate, address, and/or take measures to further prevent this from occurring. The details may or may not include names of others (students, faculty, staff). Kindly proceed in the way you feel most comfortable. To the extent possible, we will never intentionally reveal your identity. For example, you may be identifiable based on the nature of the situation, but we will protect your confidentiality in every possible way.

Ideally, how would you like the situation to be resolved? Responses may include, but are not limited to, a mediated conversation with specific colleagues/ faculty/staff, open discussion in class addressing the issue broadly without identifying information, and conversation involving only specified colleagues/faculty/ staff and leadership:

If you feel comfortable, please share any additional information that might help us to understand when and where this situation occurred (e.g., course name, study group).

(NOT REQUIRED) If you would like us to follow up with you directly, please leave your name and contact information. Your contact information will not be shared with others.

Funding No funding was provided for this project.

Data availability The data that support the findings of this study are available on request from the corresponding author.

\section{Declarations}

Conflict of interest We have no known conflicts of interest to disclose. 
Ethical approval Study-specific approval was granted from an ethics committee due to research involving humans.

Informed consent Informed consent to participate in the research was received.

\section{References}

Beaulieu, L., Addington, J., \& Almeida, D. (2019). Behavior analysts' training and practices regarding cultural diversity: The case for culturally competent care. Behavior Analysis in Practice, 12(3), 557575. https://doi.org/10.1007/s40617-018-00313-6

Behavior Analyst Certification Board. (2020). Ethics code for behavior analysts.

Behavior Analyst Certification Board. (n.d.). BACB certificant data. https://www.bacb.com/BACB-certi ficant-data

Cirincione-Ulezi, N. (2020). Black women and barriers to leadership in ABA. Behavior Analysis in Practice, 13(4), 719-724. https://doi.org/10.1007/s40617-020-00444-9

Colby, S. L., \& Ortman, J. M. (2015). Projections of the size and composition of the US population: 2014 to 2060; Population estimates and projections (Report No. 25-1143). U.S. Census Bureau.

Collis, B. (1999). Designing for differences: Cultural issues in the design of WWW-based course-support sites. British Journal of Educational Technology, 30(3), 201-216. https://doi.org/10.1111/14678535.00110

Conners, B., Johnson, A., Duarte, J., Murriky, R., \& Marks, K. (2019). Future directions of training and fieldwork in diversity issues in applied behavior analysis. Behavior Analysis in Practice, 12(4), 767776. https://doi.org/10.1007/s40617-019-00349-2

Finol, M. (2020, March 26). Asynchronous vs. synchronous learning: A quick overview. Bryn Mawr College. https://www.brynmawr.edu/blendedlearning/asynchronous-vs-synchronous-learning-quickoverview, DOI: https://doi.org/10.4103/JMAU.JMAU_49_19

Fong, E. H., Catagnus, R. M., Brodhead, M. T., Quigley, S., \& Field, S. (2016). Developing the cultural awareness skills of behavior analysts. Behavior Analysis in Practice, 9(1), 84-94. https://doi.org/10. 1007/s40617-016-0111-6

Fong, E. H., Ficklin, S., \& Lee, H. Y. (2017). Increasing cultural understanding and diversity in applied behavior analysis. Behavior Analysis: Research and Practice, 17(2), 103-113. https://doi.org/10. 1037/bar0000076

Gillborn, D. (2018). Heads I win, tails you lose: Anti-Black racism as fluid, relentless, individual and systemic. Peabody Journal of Education, 93(1), 66-77. https://doi.org/10.1080/0161956X.2017.14031 78

Glenn, S. S. (1988). Contingencies and metacontingencies: Toward a synthesis of behavior analysis and cultural materialism. The Behavior Analyst, 11(2), 161-179. https://doi.org/10.1007/BF03392470

Glenn, S. S. (2004). Individual behavior, culture, and social change. The Behavior Analyst, 27(2), 133151. https://doi.org/10.1007/BF03393175

Glenn, S. S., Malott, M. E., Andery, M. A., Benvenuti, M., Sandaker, I., Todorov, J. C., Tourinho, E. Z., \& Vasconcelos, L. A. (2016). Toward consistent terminology in a behaviorist approach to cultural analysis. Behavior and Social Issues, 25, 11-27. https://doi.org/10.5210/bsi.v25i0.6634

Gover, A. R., Harper, S. B., \& Langton, L. (2020). Anti-Asian hate crime during the COVID-19 pandemic: Exploring the reproduction of inequality. American Journal of Criminal Justice, 45(4), 647667. https://doi.org/10.1007/s12103-020-09545-1

Harvey, T. (2017). Design and pedagogy for transformative intercultural learning. In B. K. Mikk \& I. E. Seglitz (Eds.), Learning across cultures: Locally and globally (3rd ed., pp. 109-138). Stylus Publishers.

Jones, S. H., St Peter, C. C., \& Ruckle, M. M. (2020). Reporting of demographic variables in the Journal of Applied Behavior Analysis. Journal of Applied Behavior Analysis, 53(3), 1304-1315. https://doi. org/10.1002/jaba.722

Leland, W., \& Stockwell, A. (2019). A self-assessment tool for cultivating affirming practices with transgender and gender-nonconforming (TGNC) clients, supervisees, students, and colleagues. Behavior Analysis in Practice, 12(4), 816-825. https://doi.org/10.1007/s40617-019-00375-0 
Malott, M. E., \& Glenn, S. S. (2006). Targets of intervention in cultural and behavioral change. Behavior and Social Issues, 15, 31-56. https://doi.org/10.5210/bsi.v15i1.344

Maringe, F., \& Sing, N. (2014). Teaching large classes in an increasingly internationalising higher education environment: Pedagogical, quality and equity issues. Higher Education, 67, 761-782. https:// doi.org/10.1007/s10734-013-9710-0

Melendez, J. L., Tan, I. M. C., Lau, J. C., \& Leung, J. (2021). Practical resources for talking to children with autism about systemic racism. Behavior Analysis in Practice, 14, 451-461. https://doi.org/10. 1007/s40617-020-00500-4

Miller, K. L., Cruz, A. R., \& Ala'i-Rosales, S. (2019). Inherent tensions and possibilities: Behavior analysis and cultural responsiveness. Behavior and Social Issues, 28(1), 16-36. https://doi.org/10.1007/ s42822-019-00010-1

Moreno, G., \& Gaytán, F. X. (2013). Reducing subjectivity in special education referrals by educators working with Latino students: Using functional behavioral assessment as a pre-referral practice in student support teams. Emotional \& Behavioural Difficulties, 18(1), 88-101. https://doi.org/10. 1080/13632752.2012.675132

Moreno, G., Wong-Lo, M., Short, M., \& Bullock, L. M. (2014). Implementing a culturally attuned functional behavioural assessment to understand and address challenging behaviours demonstrated by students from diverse backgrounds. Emotional \& Behavioural Difficulties, 19(4), 343-355. https:// doi.org/10.1080/13632752.2013.860682

Najdowski, A. C., Gharapetian, L., \& Jewett, J. (2021). Toward the development of antiracist and multicultural graduate training programs in behavior analysis. Behavior Analysis in Practice. Advance online publication. https://doi.org/10.1007/s40617-020-00504-0

Pager, D., \& Shepherd, H. (2008). The sociology of discrimination: Racial discrimination in employment, housing, credit, asnd consumer markets. Annual Review of Sociology, 34, 181-209. https:// doi.org/10.1146/annurev.soc.33.040406.131740

Pincas, A. (2001). Culture, cognition and communication in global education. Distance Education, 22(1), 30-51. https://doi.org/10.1080/0158791010220103

Pritchett, M., Ala'i, S., Cruz, A. R., \& Cihon, T. (2020). Social justice is the spirit and aim of an applied science of human behavior: Moving from colonial to participatory research practices. https://doi. org/10.31234/osf.io/t87p4

Rispoli, M., O’Reilly, M., Lang, R., Sigafoos, J., Mulloy, A., Aguilar, J., \& Singer, G. (2011). Effects of language of implementation on functional analysis outcomes. Journal of Behavioral Education, 20(4), 224-232. https://doi.org/10.1007/s10864-011-9128-7

Sadykova, G., \& Dautermann, J. (2009). Crossing cultures and borders in international online distance higher education. Journal of Asynchronous Learning Networks, 13(2), 89-114. https://doi.org/10. 24059/olj.v13i2.1670

The Harriet W. Sheridan Center for Teaching and Learning. (n.d.). Diversity \& inclusion syllabus statements. Brown University. https://www.brown.edu/sheridan/teaching-learning-resources/inclusiveteaching/statements

The Safe Zone Project. (n.d.). The Safe Zone Project. https://thesafezoneproject.com

Wilson, D. S., Hayes, S. C., Biglan, A., \& Embry, D. D. (2014). Evolving the future: Toward a science of intentional change. The Behavioral and Brain Sciences, 37(4), 395-416. https://doi.org/10.1017/ S0140525X13001593 